\title{
CAR-TRUCK CRASHES IN THE NATIONAL MOTOR VEHICLE CRASH CAUSATION SURVEY
}

\author{
Ronald R. Knipling \\ Safety for the Long Haul Inc. \\ Arlington, Virginia, USA \\ rknipling@verizon.net
}

\begin{abstract}
Summary: The National Motor Vehicle Crash Causation Survey (NMVCCS) provided in-depth investigative data on pre-crash factors and other characteristics of 5,471 crashes involving light passenger vehicles ("cars"). Within the dataset, 199 crashes, representing 79,721 crashes nationally, were collisions between cars and large trucks. These 199 car-truck crashes constitute the second largest U.S. truck in-depth crash investigation dataset ever compiled, but its findings have not previously been published. NMVCCS is a significant source of information about the genesis of car-truck crashes. This includes variables relating to crash configurations, critical reasons, associated factors, and conditions of occurrence. Findings supplement and generally corroborate those from the Large Truck Crash Causation Study. However, NMVCCS data are more recent and represent a wider range of crash severities. Cars were more likely than trucks to be the encroaching/precipitating vehicle in car-truck collisions. Overall, 71.0\% of assigned Critical Reasons (CRs) were to the car. Cars were more likely to be outof-control prior to impact and to violate rights-of-way. Associated, contributing factors relating to driver impairment or stress were noted more frequently for car drivers. Trucks were more likely to be assigned vehicle-related CRs and associated factors, however. Nationally, about $80 \%$ of truck-related fatalities occur in car-truck crashes. Understanding their genesis is essential for the development of effective countermeasures.
\end{abstract}

\section{INTRODUCTION}

In $2012,80 \%$ of 3,921 truck crash-related fatalities occurred in multi-vehicle crashes; of these, $91 \%$ were occupants of the other vehicle (FMCSA, 2014). The motoring public pays a high toll from crashes involving large trucks, and the burden on the trucking industry is also high. Given their human and economic impact, it is essential to understand how and why these crashes happen. The Large Truck Crash Causation Study (LTCCS) remains the most extensive in-depth investigation into large truck crash genesis (FMCSA 2006). Yet LTCCS data has its limitations. The LTCCS investigated only the most serious $\sim 11 \%$ of large truck crashes, those classified by the police as either $\mathrm{K}$ (person killed in the crash), A (incapacitating injury), or B (nonincapacitating) in the "KABCO" police-reported crash severity scale. In addition, LTCCS data collection ended on December 31, 2003, four days before the onset of markedly changed truck driver Hours-of-Service (HOS) rules which, among other changes, required two additional offduty hours daily (Knipling, 2009). While the LTCCS sample of 967 crashes (492 of which were two-vehicle) exceeds any other in-depth truck study, it still does not provide final answers to many causation questions. Supplemental information sources are helpful. 
The NMVCCS, conducted by NHTSA between 2005 and 2007, reported in-depth investigations of 5,471 crashes, all involving light passenger vehicles. Of those, 199 involved both a light passenger vehicle and a large truck (NHTSA, 2008). The NMVCCS investigated no crashes occurring between midnight and 6 a.m., but, importantly for trucks, it was conducted after the major HOS changes implemented in early 2004. Most notably, the NMVCCS adds to our knowledge of large truck crash causation because it included police-reported crashes of all "KABCO" severity levels. In addition to the K, A, and B levels, it included C (possible injury) and $\mathrm{O}$ (no injury) crashes which, together, constitute the majority of police-reported crashes.

This paper reviews key causation-related variables describing these 199 car-truck crashes. It focuses on relative "fault" and on driver errors and other failures precipitating the crashes. For simplicity, medium and heavy trucks, including both single-unit and combination-unit trucks of greater than 10,000lb. Gross Vehicle Weight Rating (GVWR), are simply called "trucks." Light passenger vehicles, including cars, SUVs, light trucks, and vans, are simply called "cars."

\section{NMVCCS DATA COLLECTION AND CURRENT ANALYSIS METHODOLOGY}

To qualify for NMVCCS, a crash had to involve at least one light passenger vehicle which was towed due to collision damage, and an emergency medical squad had to be dispatched to the crash. This is a higher severity threshold than in Police Accident Report (PAR) based datasets such as the General Estimates System (GES). On the other hand, the severity threshold was lower than that of the LTCCS, which required a K, A, or B injury. Because NMVCCS investigators were quickly "on scene," they had relatively undisturbed access to crash evidence and were generally able to discuss the circumstances of the crash there with drivers, passengers, and witnesses (NHTSA, 2008). The most important causation variable discerned was the Critical Reason (CR), the salient proximal cause of the crash. As in the LTCCS, the CR was assigned to just one vehicle (or its driver) in multi-vehicle crashes. Approximately 600 other NMVCCS variables captured crash conditions of occurrence, other pre-crash events, and associated factors.

The LTCCS and NMVCCS used different criteria for designating associated factors. LTCCS factors merely had to be present to be coded (FMCSA, 2006). In the NMVCCS, an associated factor was one which was "likely to add to the probability of crash occurrence ... " (NHTSA, 2008). In other words, NMVCCS associated factors were contributory, not just present.

The need for on-scene investigations limited the NMVCCS sample to only those crashes occurring between 6 a.m. and midnight (NHTSA, 2008). In the LTCCS, 13\% of multi-vehicle truck crashes occurred during these overnight hours (Knipling and Bocanegra, 2008). The exclusion of overnight crashes did not greatly reduce the number of car-truck crashes, but it did result in an underrepresentation of factors associated with overnight driving, in particular driver fatigue (for both car and truck drivers) and alcohol use (for car drivers).

To generate nationally representative estimates, the NMVCCS used multi-stage probability sampling based on geography and urbanization. The 24 sampling locations and general methodologies were the same as those in the LTCCS. Within the sampling frame, the selection of specific crashes for investigation was random. Crash case weights permitting national estimates were inversely related to selection fractions (NHTSA, 2008). 
NMVCCS car-truck crashes are the subject of this study. For most specifications, two numbers are provided: the raw number of cases (out of 199) meeting the criteria and the weighted crash percentage based on a denominator of 79,721. All NMVCCS percentages cited here are weighted values. The percentages do not correspond directly to case counts because individual cases can have markedly different national weights. Consistent with NHTSA practice in other NMVCCS reports, and most traffic crash reporting, sampling errors are not provided. The reader should be aware that weighted national estimates and their percentages are subject to such errors. The analysis includes no statistical tests of observed differences because its goal is not to identify statistically significant differences (a low bar), but rather more pronounced differences (or the lack thereof) having clear implications regarding crash causation and countermeasures.

\section{RESULTS}

Car-truck crash conditions of occurrence were compared to those of all two-vehicle crashes; only notable differences are cited here. More than half of car-truck crashes $(52.1 \%)$ occurred on divided highways; for all two-vehicle crashes the percentage was 30.6\%. Car-truck crashes were much more likely to occur in a non-junction section of roadway (62.5\% vs. $21.7 \%)$. A far greater percentage of car-truck crashes occurred in highway work zones $(12.8 \%)$ than was the case for two-vehicle crashes in general $(2.9 \%)$.

The variable First Harmful Event Crash Type describes the action and/or relative position of each vehicle in the crash. Various rear-end crash involvement types (e.g., striking, struck) were most frequent and represented about one-third of both car and truck involvements. Figure 1 is a histogram illustrating marked car-truck differences for four selected specific crash roles. Cars were more likely than trucks to be the striking vehicle in both lead-vehicle stopped and leadvehicle moving rear-end crashes. Trucks were overinvolved in same direction sideswipes or

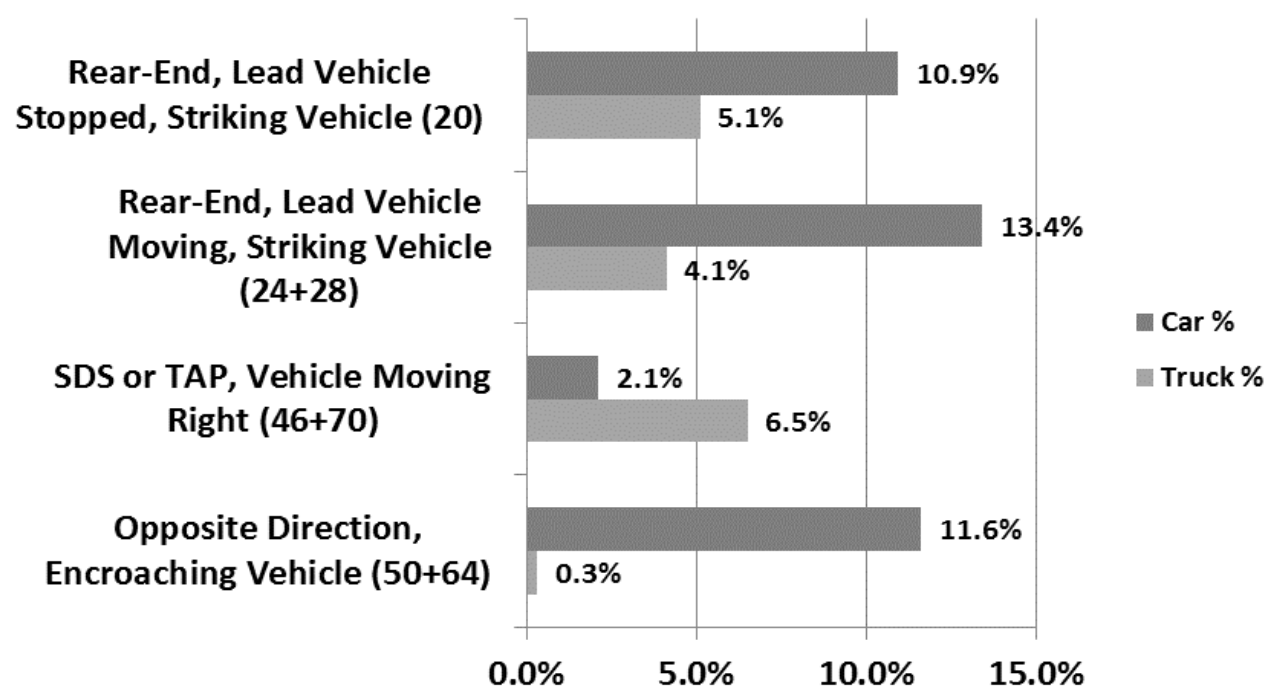

Figure 1. Selected contrasting car $\&$ truck crash role weighted percentages (within each vehicle type) SDS = Same Direction Sideswipe, TAP $=$ Turn Across Path 
turns across path when they were moving toward the right. In opposite direction crashes (i.e., head-on or opposite direction sideswipes), cars were far more likely to be the encroaching vehicle. For most other crash roles, car and truck involvement patterns were similar.

The Critical Reason (CR) is "the immediate reason for this event and is often the last failure in the causal chain" leading to the crash (NHTSA, 2008). Most CRs represent specific proximal driver errors or other failures triggering crash sequences. Table 1 shows percentages of all CRs (one assigned for each crash). They may be compared both horizontally and vertically. Altogether, cars were assigned $71.0 \%$ of CRs versus $29.0 \%$ for trucks. To find the approximate weighted number of crashes represented by each category, multiply percentages by 79,721 .

Table 1. Critical Reason groupings; case counts and weighted percentages

\begin{tabular}{|c|c|c|c|c|}
\hline Critical Reason & C\# & CW\% & $\mathbf{T} \#$ & TW\% \\
\hline Sleep, that is, actually asleep & 9 & $\mathbf{5 . 5 \%}$ & $\mathbf{0}$ & $0.0 \%$ \\
\hline $\begin{array}{l}\text { Heart Attack, Other Physical Impairment, Other Critical } \\
\text { Non-Performance }(101+102)\end{array}$ & 5 & $6.7 \%$ & $\mathbf{0}$ & $0.0 \%$ \\
\hline Inattention (i.e. Daydreaming) & 6 & $2.0 \%$ & $\mathbf{0}$ & $0.0 \%$ \\
\hline Internal Distraction & 20 & $6.6 \%$ & 5 & $0.7 \%$ \\
\hline External Distraction & 4 & $1.5 \%$ & 2 & $0.3 \%$ \\
\hline $\begin{array}{l}\text { Inadequate Surveillance (e.g. Failed to Look, Looked But Did } \\
\text { Not See) }\end{array}$ & 19 & $7.3 \%$ & 17 & $6.1 \%$ \\
\hline Other or Unknown Recognition Error (114+119) & 4 & $2.2 \%$ & 3 & $1.7 \%$ \\
\hline $\begin{array}{l}\text { Too Fast for Conditions, to be able to respond to unexpected } \\
\text { actions, or for curve/turn }(120+121+122)\end{array}$ & 8 & $10.9 \%$ & 3 & $0.5 \%$ \\
\hline Misjudgment of Gap or Other's Speed & 6 & $1.0 \%$ & 6 & $\mathbf{2 . 8 \%}$ \\
\hline Following too Closely to Respond to Unexpected Actions & 1 & $0.2 \%$ & 1 & $0.7 \%$ \\
\hline False Assumptions of Other Road User's Actions & $\mathbf{5}$ & $2.2 \%$ & 8 & $4.3 \%$ \\
\hline Illegal Maneuver & 7 & $1.5 \%$ & 6 & $0.7 \%$ \\
\hline $\begin{array}{l}\text { Inadequate or Incorrect Evasive Action, e.g. Braking Only, } \\
\text { Not Braking and Steering }(129+130)\end{array}$ & 6 & $3.0 \%$ & $\mathbf{0}$ & $0.0 \%$ \\
\hline Aggressive Driving Behavior & 4 & $1.3 \%$ & $\mathbf{0}$ & $0.0 \%$ \\
\hline Turned with Obstructed View & 1 & $0.1 \%$ & 1 & $0.9 \%$ \\
\hline Other or Unknown Decision Error $(132+139)$ & 3 & $1.1 \%$ & 1 & $\mathbf{0 . 0 \%}$ \\
\hline Overcompensation & 4 & $\mathbf{0 . 5 \%}$ & $\mathbf{0}$ & $\mathbf{0 . 0 \%}$ \\
\hline $\begin{array}{l}\text { Poor Directional Control e.g., Failing to Control Vehicle with } \\
\text { Skill Ordinarily Expected }\end{array}$ & 3 & $0.6 \%$ & 4 & $0.7 \%$ \\
\hline Unknown Performance Error & $\mathbf{0}$ & $0.0 \%$ & 1 & $0.0 \%$ \\
\hline Type of Driver Error Unknown & 10 & $16.6 \%$ & 3 & $0.3 \%$ \\
\hline $\begin{array}{l}\text { Vehicle Component Failure (e.g., Brakes, Tires) } \\
(200+202+211+213)\end{array}$ & $\mathbf{0}$ & $\mathbf{0 . 0 \%}$ & 5 & $2.6 \%$ \\
\hline Vehicle-Related Vision Obstructions & $\mathbf{0}$ & $0.0 \%$ & 3 & $4.6 \%$ \\
\hline Cargo Shifted & $\mathbf{0}$ & $0.0 \%$ & $\mathbf{1}$ & $1.9 \%$ \\
\hline Environmental (e.g., Slick Roads, Glare) $(509+525)$ & $\mathbf{2}$ & $0.3 \%$ & $\mathbf{0}$ & $\mathbf{0 . 0 \%}$ \\
\hline Unknown Reason for Critical Event & 2 & $0.0 \%$ & 1 & $0.1 \%$ \\
\hline Total, Critical Reason Assigned to this Vehicle & 128 & $71.0 \%$ & 71 & $29.0 \%$ \\
\hline
\end{tabular}

One sees in Table 1 that cars were most overrepresented in the following CRs: asleep-at-thewheel, physical impairments, inattention and distraction, excessive speed, and unknown driver errors. Inadequate surveillance was among the top CRs for both. Trucks were overrepresented in false assumptions and vehicle-related factors. Figure 2 is a histogram showing percentages for each vehicle type for several contrasting CRs. Additional groupings in the figure include critical 
non-performance (sleep + heart attack or other physical impairment), inattention and distraction (both internal and external), and vehicle factors (component + vision obstruction + cargo shift).

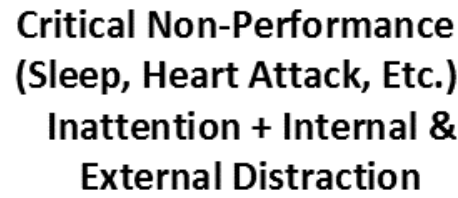

Too Fast

Type of Driver Error Unknown

False Assumption

Vehicle-Related

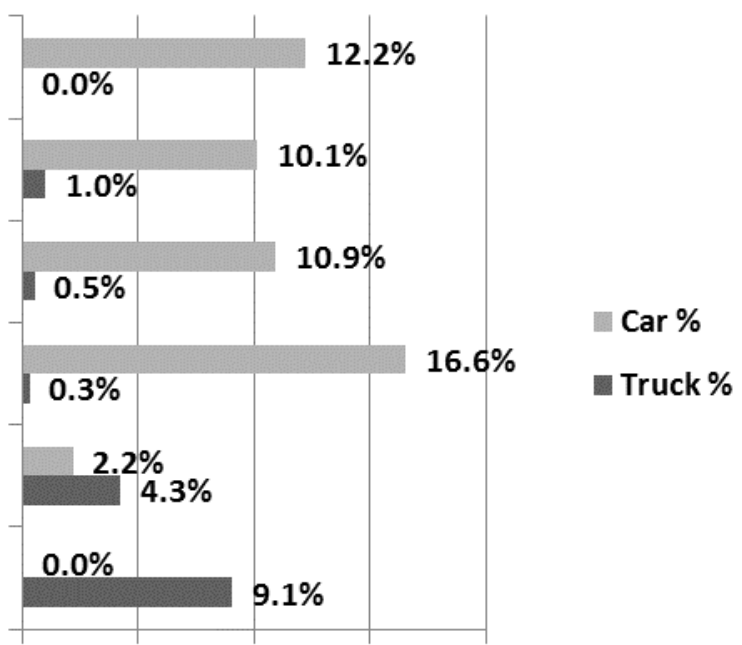

$0.0 \% \quad 5.0 \% \quad 10.0 \% 15.0 \% 20.0 \%$

Figure 2. Car and truck weighted percentages (of all crashes) for CR groupings

Pre-Impact Stability and Right-of-Way Violation are two additional causal variables. Cars were about twice as likely as trucks to be out-of-control (i.e., skidding or otherwise not tracking) prior to their impacts; $36(18.9 \%)$ were so coded. This compares to 18 trucks $(7.7 \%)$. Cars were also more likely to violate rights-of-way. Among cars with known right-of-way (i.e., coded yes or no), 57.9\% did not have the right-of-way. This contrasts with $39.3 \%$ of trucks.

Table 2 provides comparative car-truck unweighted case numbers and weighted percentages for seven associated (i.e., contributing) factors, most relating to driver impairment and stress. These factors were not mutually exclusive; more than one could be coded for any driver/vehicle. For all measures, more car drivers were impaired and/or under stress than truck drivers. For impairment (fatigued, ill, alcohol), car drivers were assigned a total of 36 codes, versus 8 for truck drivers. For stress (aggression, work pressure, hurry), car drivers received 30 codes compared to 11 for truck drivers. Trucks were much more likely to have a crash-contributing vehicle-related condition, however.

Table 2. Associated factors for cars \& trucks; case numbers (of 199) and weighted percentages

\begin{tabular}{lrrrr}
\hline Associated Factor: & C\# & CW\% & T\# & TW\% \\
\hline Driver Fatigued & $\mathbf{2 3}$ & $\mathbf{1 6 . 6 \%}$ & $\mathbf{5}$ & $\mathbf{1 . 9 \%}$ \\
\hline Driver Ill (Medically Verified) & 7 & $\mathbf{3 . 4 \%}$ & $\mathbf{3}$ & $\mathbf{1 . 7 \%}$ \\
\hline Police-Reported Alcohol & $\mathbf{6}$ & $\mathbf{1 . 0 \%}$ & $\mathbf{1}$ & $\mathbf{0 . 3 \%}$ \\
\hline Driver Aggressive Acts & $\mathbf{9}$ & $\mathbf{3 . 1 \%}$ & $\mathbf{0}$ & $\mathbf{0 . 0} \%$ \\
\hline Work-Related Stress/Pressure & $\mathbf{9}$ & $\mathbf{1 3 . 5 \%}$ & $\mathbf{4}$ & $\mathbf{1 . 6 \%}$ \\
\hline Driver in a Hurry & $\mathbf{1 2}$ & $\mathbf{2 . 8 \%}$ & $\mathbf{7}$ & $\mathbf{0 . 8 \%}$ \\
\hline Vehicle-Related Condition & 12 & $\mathbf{3 . 3 \%}$ & $\mathbf{2 0}$ & $\mathbf{1 2 . 0} \%$ \\
\hline
\end{tabular}




\section{DISCUSSION}

NMVCCS car-truck crash statistics have corroborated previous studies (e.g., Starnes, 2006; FHWA, 1999; Moonesinghe et al, 2003; Kostyniuk et al 2002) finding car drivers to be principally responsible for precipitating most of car-truck crashes. The present car-CR percentage of $71.0 \%$ is similar to past studies of fatal crashes (e.g., Blower, 1998). What is most different here is that NMVCCS cases included all police-reported KABCO levels.

Particular scenarios seem to dominate car-truck crash statistics. As in other data (e.g., FMCSA, 2014; Moonesinghe et al, 2003), cars were most likely to be the encroaching vehicle in rear-end and opposite direction (e.g., head-on) crashes. Car drivers were more likely to be asleep, otherwise impaired (by fatigue, illness, or alcohol), inattentive, speeding, aggressive, and/or in a hurry than were truck drivers. Cars were more likely to be out-of-control prior to impact and to violate rights-of-way.

Driver fatigue dominates many discussions of truck safety, in large part due to long hours driven by many truck drivers. It may seem paradoxical that car drivers were far more likely to be asleep or fatigued in NMVCCS car-truck crashes, but the finding is entirely consistent with LTCCS and other past reports (Starnes, 2006; Kostyniuk et al 2002). In LTCCS car-truck crashes, the ratio of car drivers asleep to truck drivers asleep was 8:1 (Knipling, 2009). The NMVCCS excluded overnight (midnight to 6 a.m.) crashes, but this exclusion likely affected car and truck drivers similarly. Also notable is the fact that here, as in other studies, car drivers seem more likely to be fatigued (or ill) in their crashes with trucks than in their crashes overall. Perhaps the overinvolvement of trucks in car-driver-impairment crashes is simply related to their size as a random "target" and/or the limited capabilities of trucks to make evasive maneuvers.

Recall the high percentage of car-truck crashes occurring in highway work zones $(12.8 \%)$, compared to just $2.9 \%$ of other NMVCCS two-vehicle crashes $(2.9 \%)$. Similarly, $13 \%$ of all LTCCS crashes occurred in work zones. Knipling (2009) has estimated that large truck crash risk (vis-à-vis exposure) may be elevated as much as 20 -fold in work zones. Trucks' large size seems to interact with constricted spaces in work zones to heighten crash risk.

In spite of small-sample concerns, this study's findings affirm the value of crash causation information for identifying and designing truck crash countermeasures. The high incidence of truck-struck rear-end crashes $(24.5 \%$ of the total) suggest the value of enhanced conspicuity treatments or active warning systems on the rear of trucks and trailers. Trucks were relatively most likely to encroach other vehicles when moving laterally from left to right, attesting to their need for better right side visibility. Trucks were assigned vehicle-related CRs in $9.1 \%$ of all the crashes, and there were additional crashes where truck vehicle factors contributed. This supports the need for vehicle-related regulations and enforcement, but it is also a reminder that most truck crashes are not primarily related to vehicle factors. Most crashes, whether precipitated by the car or by the truck, are due to driver mistakes and misbehaviors.

Leonard Evans has noted that "A large portion of truck safety is rooted in the context of overall traffic safety" (Evans, 2001). Study findings validate this view. Most truck crashes involve cars. 
Car drivers precipitate most of these crashes and their causes, with some exceptions as noted here, are much like those of motor vehicle crashes in general. Nationally and internationally, cartruck crashes may best be reduced not by truck safety initiatives per se but rather by broader efforts to improve the safety performance of all drivers.

\section{ACKNOWLEDGMENTS}

The author thanks the staff of the NHTSA National Center for Statistics and Analysis, including Seymour Stern, Adam Toth, and Lyn Cianflocco, for retrieving the NMVCCS data in this paper per specifications provided by author. Additional statistics on NMVCCS car-truck crashes not published here are available from NHTSA or from the author.

\section{REFERENCES}

Blower, D.F. The Relative Contribution of Truck Drivers and Passenger Car Drivers to TwoVehicle, Truck-Car Traffic Crashes. Publication No. UMTRI-98-25, UMTRI, Ann Arbor, MI. 1998.

Evans, L. (2001) Major factors relating to traffic safety. Conference on Improving Safety for Drivers and Fleets: Historical and Innovative Approaches, Carnegie Mellon University, Pittsburgh, June 18-19, 2001.

Federal Highway Administration Office of Motor Carriers \& Highway Safety. (1999) Driverrelated factors in crashes between large trucks and passenger vehicles, Analysis Brief MCRT-99-011. Washington, DC: Department of Transportation.

FMCSA. (2006) Report to Congress on the Large Truck Crash Causation Study (LTCCS). Washington, DC: Department of Transportation.

FMCSA. (2014) Large truck crash facts 2012. FMCSA-RRA-14-004, ). Washington, DC: Department of Transportation.

Knipling, R.R. \& J. Bocanegra. (2008) Comparison of Combination-Unit Truck and Single-Unit Truck Statistics from the LTCCS. FMCSA \& Volpe Center. Project report. 2008.

Knipling, R.R. (2009) Safety for the long haul; large truck crash risk, causation, \& prevention. Arlington, Virginia: American Trucking Associations. ISBN 978-0-692-00073-1.

Kostyniuk, L. P., F. M. Streff, and J. Zakrajsek. (2002) Identifying unsafe driver actions that lead to fatal car-truck crashes. Washington, DC: AAA Foundation for Traffic Safety.

Moonesinghe, R., A. Longthorne, U. Shankar, S. Singh, R. Subramanian, and J. Tessmer. (2003) An analysis of fatal large truck crashes, NHTSA Publication DOT HS 809 569, Washington, DC: Department of Transportation.

NHTSA. (2008) National Motor Vehicle Crash Causation Survey (Report to Congress, July 2008). DOT HS 811 059, Washington, DC: Department of Transportation.

Starnes, M. (2006) LTCCS: an initial overview. NHTSA Publication DOT HS 810 646, NHTSA, Washington, DC: Department of Transportation. 